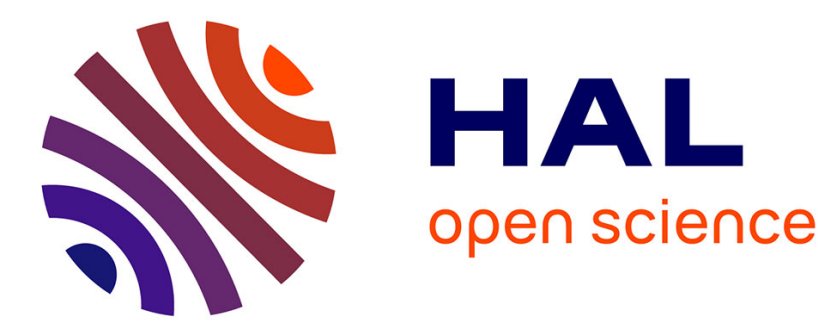

\title{
De l'hospitalisation aux soins sans consentement en psychiatrie \\ Livia Velpry
}

\section{To cite this version:}

Livia Velpry. De l'hospitalisation aux soins sans consentement en psychiatrie. Journal français de psychiatrie, 2011, 38, pp.16-18. 10.3917/jfp.038.0016 . halshs-00909674

\section{HAL Id: halshs-00909674 \\ https://shs.hal.science/halshs-00909674}

Submitted on 23 Feb 2016

HAL is a multi-disciplinary open access archive for the deposit and dissemination of scientific research documents, whether they are published or not. The documents may come from teaching and research institutions in France or abroad, or from public or private research centers.
L'archive ouverte pluridisciplinaire HAL, est destinée au dépôt et à la diffusion de documents scientifiques de niveau recherche, publiés ou non, émanant des établissements d'enseignement et de recherche français ou étrangers, des laboratoires publics ou privés. 


\title{
DE L'HOSPITALISATION AUX SOINS SANS CONSENTEMENT EN PSYCHIATRIE
}

\author{
Livia Velpry
}

ERES | Journal français de psychiatrie

\author{
$2011 / 1-n^{\circ} 38$ \\ pages 16 à 18
}

ISSN 1260-5999

Article disponible en ligne à l'adresse:

http://www.cairn.info/revue-journal-francais-de-psychiatrie-2011-1-page-16.htm

Pour citer cet article :

Velpry Livia, « De I'hospitalisation aux soins sans consentement en psychiatrie », Journal français de psychiatrie, 2011/1 n³8, p. 16-18. DOI : 10.3917/jfp.038.0016

Distribution électronique Cairn.info pour ERES.

(C) ERES. Tous droits réservés pour tous pays.

La reproduction ou représentation de cet article, notamment par photocopie, n'est autorisée que dans les limites des conditions générales d'utilisation du site ou, le cas échéant, des conditions générales de la licence souscrite par votre établissement. Toute autre reproduction ou représentation, en tout ou partie, sous quelque forme et de quelque manière que ce soit, est interdite sauf accord préalable et écrit de l'éditeur, en dehors des cas prévus par la législation en vigueur en France. II est précisé que son stockage dans une base de données est également interdit. 


\section{De l'hospitalisation aux soins sans consentement en psychiatrie \\ Livia Velpry*}

\begin{abstract}
Une loi relative aux droits et à la protection des personnes faisant l'objet de soins psychiatriques et aux modalités de leur prise en charge a été promulguée le 5 juillet $2011^{1}$. Dans le texte de cette nouvelle loi, qui reprend la structure de la loi précédente $^{2}$, l'expression « hospitalisation sans consentement» a été remplacée par celle de "soins sans consentement $»$. Cette modification sémantique étend de fait la possibilité de l'intervention sans consentement à tous les modes de prise en charge en psychiatrie, notamment ambulatoire ${ }^{3}$. D'une certaine manière, on peut considérer que cette substitution s'inscrit dans la logique des transformations qu'a connues l'institution psychiatrique au cours du $\mathrm{XX}^{\mathrm{e}}$ siècle. Depuis la loi de 1838 régissant l'internement psychiatrique au sein de l'asile, alors unique lieu de soin, le dispositif de soins psychiatrique s'est diversifié en de multiples lieux et modes de prise en charge. Pourquoi, alors, cantonner les possibilités d'intervention thérapeutique sans le consentement de la personne au cadre de l'hôpital ? La question est d'autant plus pertinente qu'en France, depuis plusieurs décennies, des lois encadrent certaines interventions auparavant réalisées à l'asile sous le régime de la loi de 1838 , sous la forme de mesures de protection des personnes et de biens particulièrement ${ }^{4}$.

Cette contribution propose tout d'abord de revenir sur les arguments soulevés par la mise en place de mesures du même type dans d'autres pays. En effet, de telles modalités thérapeutiques, obligeant la personne à suivre un protocole de soin hors procédure pénale, et reposant sur une décision du juge - ou de l'autorité administrative-, existent
\end{abstract}

\footnotetext{
* Sociologue, maître de conférences à l'université Paris VIII.
}

sous diverses formes, la conception des mesures variant notamment selon le système légal et psychiatrique propre à chaque pays. Aux États-Unis, par exemple, ces mesures se rencontrent dans de nombreux États ${ }^{5}$, dans certains cas depuis les années 1970. C'est également le cas en Australie, en Nouvelle-Zélande, en Israël, au Royaume-Uni, en Écosse et en Suisse. Dans un second temps, nous chercherons à mieux comprendre quels usages cliniques sont effectivement faits de ces mesures et quelles difficultés spécifiques elles soulèvent. Pour cela, nous nous appuyons sur un corpus de rapports et d'articles publiés dans des revues françaises et étrangères de psychiatrie et de sciences sociales.

\section{Les termes \\ d'un débat récurrent}

Le principe de soins sans consentement en dehors de l'hôpital comme les conditions de leur mise en œuvre continuent de faire débat dans les pays qui les ont instaurés, et ce parfois depuis plus de trente ans. Ces discussions ne remettent pas en cause, d'ailleurs, l'existence de telles mesures. Quels sont les termes de ces débats?

Le principal argument des détracteurs de ce type de mesure
1. Loi no $2011-803$ du 5 juillet 2011 relative aux droits et à la protection des personnes faisant l'objet de soins psychiatriques et aux modalités de leur prise en charge, JORF $\mathrm{n}^{\circ} 155$ du 6 juillet 2011.

2. Loi n ${ }^{\circ} 90-527$ du 27 juin 1990 relative aux droits

et à la protection des personnes hospitalisées en raison de troubles mentaux

et à leurs conditions d'hospitalisation, $J O R F \mathrm{n}^{\circ} 150$ du 30 juin 1990.

3. Voir P. Rhenter, L. Velpry,

«La réforme

des hospitalisations

sans consentement », Regards sur l'actualité,

La Documentation française,

$n^{\circ} 374$, octobre 2011 .

4. Voir B. Eyraud, «Protection de la personne et soins psychiatriques : des réformes législatives peu coordonnées », Regards sur l'actualité,

La Documentation française, n' 374, octobre 2011. 5. Voir Brazelton Center For Mental Health Law, Involuntary Outpatient Commitment. Summary of State Statutes, avril 2000,

http ://www.brazelton.org/Link Click.aspx ?fileticket=CBmFgy A4i-w\%3d\&tabid=324 concerne l'extension d'un contrôle social exercé par les soignants sur les personnes ayant des troubles mentaux qui vivent dans la communauté. Cette crainte est particulièrement forte lors de la mise en place des mesures, qui interviennent très fréquemment en réponse à la survenue d'actes de violence médiatisés et commis par des personnes ayant des troubles mentaux. La mesure de soins sans consentement en ambulatoire est alors dénoncée comme une mesure moins sanitaire que sécuritaire, qui est essentiellement un moyen de maintenir une surveillance des personnes au motif qu'elles présentent un risque de commettre des actes de violence.

Une fois les mesures effectivement instaurées, l'argument d'un renforcement du contrôle social perdure dans la littérature psychiatrique sous une forme différente. La critique porte ainsi plutôt sur l'inéluctable élargissement des critères pouvant justifier de la mesure, dans la loi ou dans les pratiques. En effet, les mesures de soins sans consentement reprennent généralement, au moins dans un premier temps, les mêmes critères que ceux nécessaires à une hospitalisation. C'est d'ailleurs le cas en France. Dans la nouvelle loi, une mesure de soins sans consentement est prise à l'issue d'une période d'observation de 72 heures qui se déroule en hospitalisation complète. Dans le 
cas d'une admission en soins à la demande d'un tiers, les deux conditions nécessaires sont l'impossibilité de consentir aux soins et un état nécessitant des soins immédiats assortis d'une surveillance médicale régulière. Dans le cas d'une admission en soins sur décision du représentant de l'État, au critère de la nécessité de soin s'ajoute celui de compromettre

deux vitesses » où les ressources seraient concentrées sur les patients réticents au traitement, au détriment des patients volontaires. Une telle tendance serait favorisée par un contexte de rationalisation de la gestion du dispositif de santé mentale. En retour,

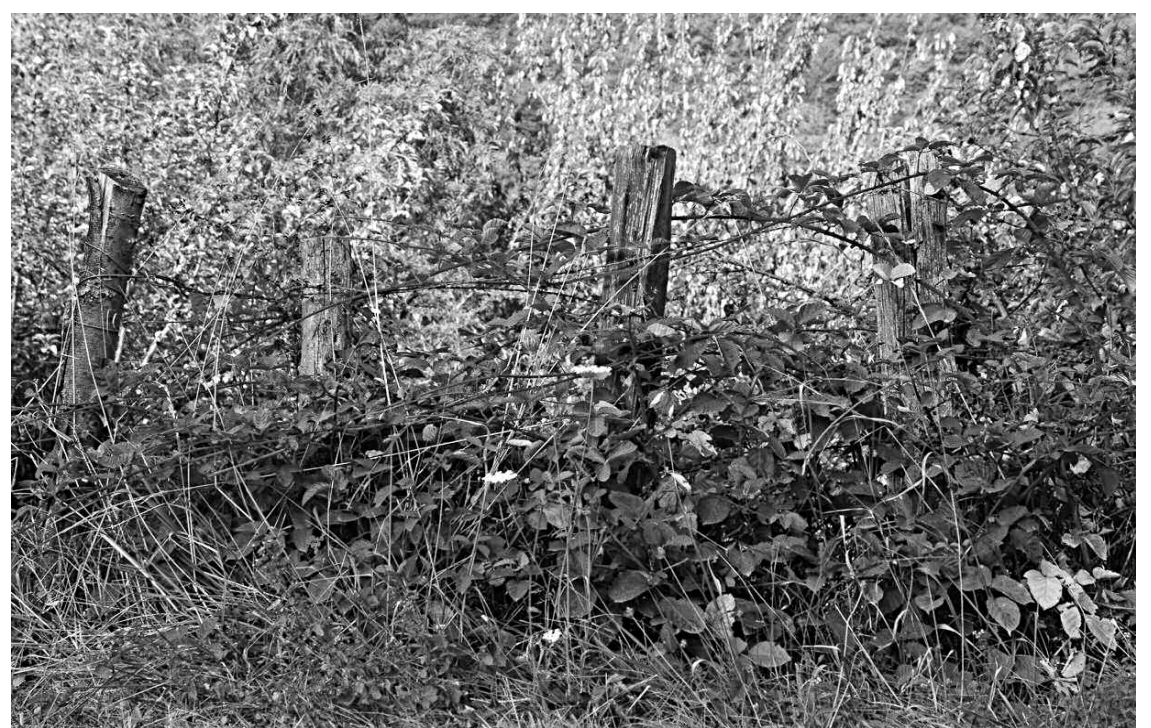

la sûreté des personnes ou de porter une atteinte grave à l'ordre public. Dans ces deux cas, les soins ambulatoires interviennent comme une alternative à une hospitalisation dans une situation où les soins sont nécessaires et doivent être imposés. Les législations des différents pays combinent elles aussi le plus couramment trois critères qui sont la dangerosité pour soi ou pour autrui, le besoin de soin immédiat et l'incapacité à consentir. Or, si le critère de dangerosité est rempli au sens le plus strict, dans quelle mesure est-il raisonnable de ne pas recourir à l'hospitalisation? À l'inverse, comment le besoin de soin immédiat peut-il être un critère pour instaurer une mesure de soin au long cours dans la communauté ? De la même manière, peut-on considérer que l'incapacité à consentir soit constante sur une longue durée pour des personnes ayant par ailleurs une vie quotidienne au moins en partie autonome? Les tenants de cette position soulignent la difficulté de déterminer de façon tranchée la capacité ou l'incapacité à consentir aux soins, critère nécessaire à l'instauration de la mesure, ce tout particulièrement dans la durée, alors que les symptômes des troubles fluctuent ${ }^{6}$. En pratique, la définition de ces critères appliqués aux soins ambulatoires sans consentement devra donc nécessairement être plus lâche, sauf à considérer qu'une partie des hospitalisations sans consentement sont abusives.

Le risque d'une extension des critères d'intervention serait de généraliser le recours à des mesures de soin sans consentement pour l'ensemble des patients réfractaires au traitement et à la psychiatrie. Il s'agit donc également, par là, de porter la discussion sur le terrain des modalités de prise en charge des troubles mentaux graves. Les nombreuses études visant à déterminer l'efficacité de ce type de mesure d'un point de vue clinique aboutissent à des résultats incertains ${ }^{7}$. Cependant, elles soulignent toutes combien il est difficile de distinguer, dans les effets de la mesure, ce qui tient à sa dimension d'obligation légale et ce qui relève plutôt du fait qu'elle s'accompagne généralement d'un suivi plus intensif des personnes. En termes de dispositif de soins psychiatriques, ces opposants soulignent alors le danger d'aboutir à un système «à par un effet pervers, les soignants pourraient être amenés à multiplier les mesures de soins sans consentement avec l'objectif d'assurer un meilleur accès aux ressources.

La réciprocité de l'obligation présente dans la mesure de soins sans consentement est, de fait, un argument mobilisé pour justifier de l'utilité de la mesure, particulièrement ces dernières années. Invoquant le réalisme et la nécessité de prendre en compte les financements souvent insuffisants $d u$ système de santé mentale, certains soulignent que l'encadrement légal des mesures fait également peser sur les pouvoir publics l'obligation de fournir les moyens de les mettre en œuvre. Au risque de la généralisation de la contrainte, les défenseurs de ces mesures opposent le bénéfice d'offrir un suivi plus intensif ${ }^{8}$. Pour certains, l'objectif assigné à un tel suivi est de réduire ou de mieux maîtriser le risque de violence que continueraient à poser certaines personnes ayant des troubles psychiatriques lorsque prend fin leur hospitalisation. Une telle conception explicitement sécuritaire a été soutenue en France, par exemple, par Jean-Louis Senon, lorsqu'il défend la nécessité de « soins ambulatoires sous la contrainte destinés aux malades mentaux ayant commis des violences ", appelés à répondre tout à la fois à « l'accumulation de pathologies psychiatriques en des malades mentaux échappant aux soins et aux problèmes posés par la prise en charge de malades mentaux est aussi régulièrement utilisé dans une tout autre perspective. Pour de nombreux psychiatres, un tel suivi a vocation à prévenir les rechutes de détention, aux violences commises par irresponsabilisés ${ }^{9} »$. Mais l'argument
6. J. Dawson, « Fault-lines in community treatment order legislation ", International Journal of Law and Psychiatry, 29, p. 482-494, 2006. 7. Voir par exemple S. Kisely, L.A. Campbell, A. Scott, N.J. Preston, J. Xiao, «Randomized and nonrandomized evidence for the effect of compulsory community and involuntary out-patient treatment on health service use : systematic review and meta-analysis ",,

Psychological Medicine, $\mathrm{n}^{\circ} 37$, 1-2007, p. 3-14.

8. Voir H. Strohl, « Le soin contraint ne peut pas remplacer l'offre adaptée de soins ", L'Information psychiatrique, $2009, n^{\circ} 85$, p. 499-507. 9. J.-L. Senon, « Soins ambulatoires sous contrainte une mise en place indispensable pour préserver une psychiatrie publique moderne ",

L'Information psychiatrique, 2005.

10. V. Hiday, « Outpatient commitment. The state of empirical research on its outcome ", Psychology, Public Policy, and Law, $\mathrm{n}^{\circ} 9(1 / 2)$, 2003, p. 8-32

11. J. Dawson, S. Romans,

A. Gibbs et N. Ratter,

« Ambivalence about community treatment orders », International Journal of Law and Psychiatry, $\mathrm{n}^{\circ} 26,2003$, p. 252

12. V. Hiday, op. cit.

13. T.L. Scheid-Cook, «Outpatient commitment as both social control and least restrictive alternative», The Sociological Quarterly, nº 32 (1), mars 1991, p. 43-60. personnes en rupture de soins. Certains pays ou États américains ont d'ailleurs intégré cette dimension dans la définition de la mesure. C'est ainsi que la Caroline du Nord a élargi le critère de la nécessité des soins à celle de prévenir la détérioration de l'état de la personne ${ }^{10}$. Avec le même objectif d'extension des critères, la législation néo-zélandaise établit que la personne doit présenter « un état mental anormal», en ajoutant que celui-ci peut être « de nature continue ou intermittente ${ }^{11} »$. Elle ouvre ainsi la possibilité de maintenir une mesure de soins sans consentement dans la durée même si les symptômes conduisant à l'incapacité de consentir fluctuent.

Dans cette perspective, la mesure de soins sans consentement dans la communauté apparaît comme une mesure préférable, car moins restrictive des libertés que le recours à l'hospitalisation. Ce faisant, les tenants de cette position reprennent un argument très influent au moment de la mise en place de ces mesures dans les années 1970 aux États-Unis. Dans une période alors influencée par les mouvements de défense des droits des patients, les soins ambulatoires étaient considérés comme moins attentatoires aux libertés individuelles et devaient donc être systématiquement préférés à l'hospitalisation, à chaque fois que c'était possible ${ }^{12}$. Dans le contexte américain des années 1990, plus marqué par la préoccupation de maîtrise des coûts des soins de santé mentale, l'avantage revendiqué de ces mesures est plutôt de permettre de diminuer le recours à l'hospitalisation dans le cas de patients dits « chroniques » qui sont hospitalisés de façon répétée ${ }^{13}$. Avec ce déplacement, les mesures de soins sans consentement sont situées au cœur des discussions portant sur la conception du dispositif de prise en charge en santé mentale pour les personnes souffrant de troubles mentaux graves. Qu'en est-il de l'usage de ces mesures et de leur place dans l'organisation des soins?

\section{Des usages des mesures aux critères}

Le recours aux mesures de soins ambulatoires sans consentement est extrêmement variable localement. Une même mesure peut ainsi être totalement intégrée aux outils thérapeutiques dans un centre de soins psychiatriques où elle sera fréquemment utilisée, et n'être jamais mobilisée dans un centre voisin. Ce constat est fait par exemple dans le 
cas des différents comtés que comprend l'État de New York ${ }^{14}$ ou entre les régions de NouvelleZélande. Il appelle à examiner de plus près les usages que font les psychiatres d'une mesure qui reste essentiellement sanitaire ${ }^{15}$ et qui s'inscrit dans un ensemble de pratiques cliniques.

Envisager le recours à des soins sans consentement dans l'ensemble des pratiques de soin nécessite de tenir compte de la difficulté à définir de façon tranchée la frontière entre soins consentis et soins obligés dans les pratiques effectives, particulièrement en dehors de l'hôpital. Cette difficulté se traduit dans la diversité des cadres, des degrés et de la nature de la contrainte exercée sur les personnes afin de les maintenir dans une relation de soin ${ }^{16}$. En France, par exemple, les sorties d'essai sont depuis longtemps utilisées, dans certains secteurs, afin d'obliger certains patients à poursuivre un suivi ambulatoire. Avec ce type de mesure, courant également dans d'autres pays sous le terme de « sorties conditionnelles » (conditional release), la personne conserve le statut de patient hospitalisé sans consentement alors qu'elle est physiquement sortie de l'hôpital. Est ainsi maintenue ouverte la possibilité de réintégrer l'hôpital en cas de non-respect du programme de soin, sans procédure légale particulière. À cet égard, l'introduction d'une mesure de soins ambulatoires sans consentement peut avoir comme effet «d'améliorer la formalisation et la transparence ${ }^{17} »$ du processus. Il existe également à l'étranger des programmes de soins ambulatoires dits «agressifs » ou « intensifs » qui mobilisent diverses pressions informelles pour maintenir les patients dans un suivi. Ce type de programme s'appuie sur des incitations, voire des menaces, portant

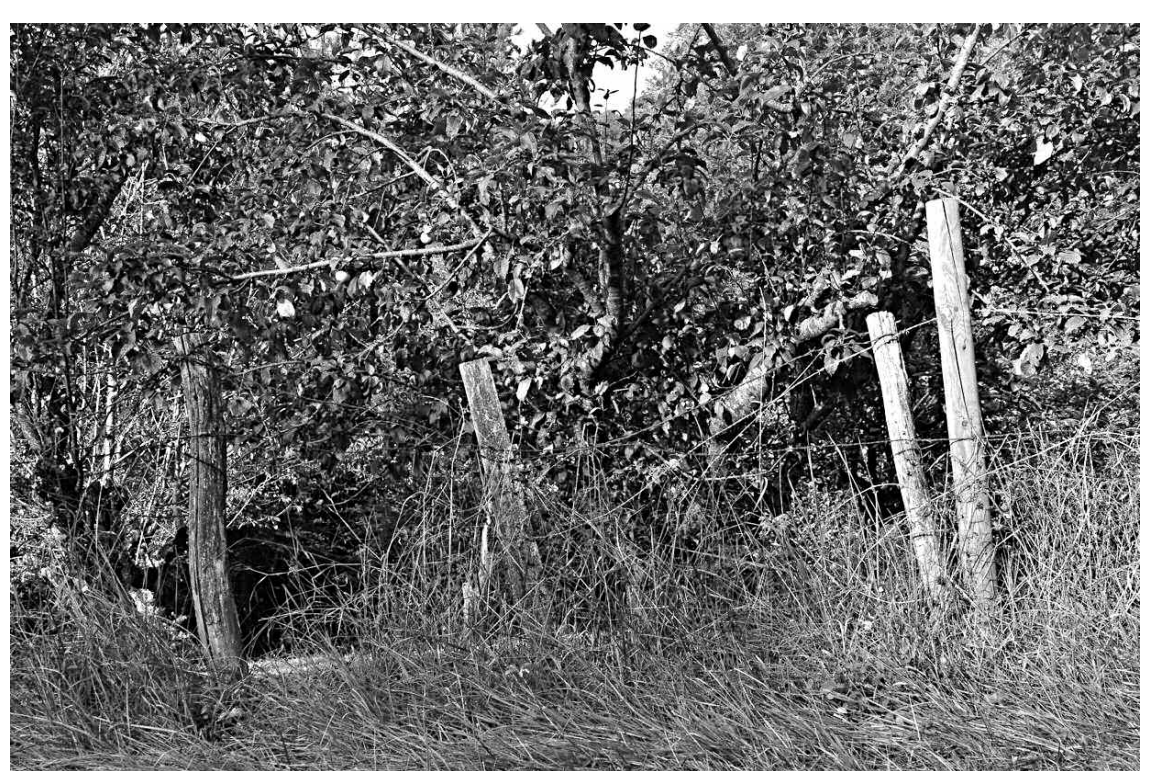

notamment sur l'accès à des ressources. Il peut donc faire peser sur la personne une contrainte tout à fait réelle à se maintenir dans des soins, même s'il ne repose pas sur une obligation de soin formalisée.

Ces réflexions soulèvent des questions éthiques plus générales sur la relation entre soignant et patient, toujours prise dans une tension entre protection de la personne et respect de ses droits. D'une manière générale, aucune des études menées sur la mise en œuvre des mesures de soins sans consentement ne conforte l'objection parfois mobilisée par les détracteurs de ces mesures, celle de l'impossibilité de fonder l'alliance thérapeutique nécessaire à des soins dans la communauté, lorsque les soins sont contraints. L'enjeu de la discussion porte bien plus sur les dynamiques de la contrainte dans la relation thérapeutique, qui nécessitent une analyse fine ${ }^{18}$. La relation de soins en psychiatrie se déploie souvent au sein d'un ensemble de « prises en charge non consensuelles ${ }^{19}$ » qui mobilisent divers moyens de pression allant de la persuasion à l'hospitalisation sans consentement.

Dans cet ensemble, où la contrainte est plus ou moins formalisée et sanctionnée légalement, les mesures de soins ambulatoires sans consentement apparaissent comme un des outils permettant de faire " levier" pour amener la personne à fréquemment celui d'accepter d'être suivie et de prendre son traitement ${ }^{20}$. Une recherche qualitative menée en Caroline du Nord ${ }^{21}$ montre que les cliniciens comme les patients engagés dans des mesures de soins sans consentement perçoivent effectivement cette mesure comme un levier supplémentaire à la disposition des soignants. Les mesures de soins distinguent par le fait qu'elles formalisent une obligation de suivi dans un cadre non hospitalier. Cette formalisation peut alors être considérée comme plus adaptée à leur usage clinique effectif par exemple que le régime des sorties d'essai, et souhaitable à ce titre $^{22}$. D'autres procédures, qui

répondent à des situations où le consentement aux soins fait défaut ou problème, peuvent être intégrées à ce que certains auteurs américains qualifient de mesures de « prises en charge assistées ${ }^{23} »$. On citera notamment les mesures de protection de la personne (tutelle, curatelle) qui, dans certains pays, permettent d'obliger au soin, mais également les directives avancées, document contractuel établi avec un soignant qui permet à une personne d'exprimer ses volontés à l'avance, dans l'éventualité de survenue d'une situation où elle ne serait plus capable de consentir. un objectif souhaité, en l'occurrence ambulatoires sans consentement se
14. M.S. Swartz, J.W. Swanson,

P.C. Robbins, H.J. Steadman, J. Monahan, New York State assisted Outpatient Treatment Program Evaluation, Duke

University School of Medicine, Durham, NC, juin 2009 ;

J. Dawson, « Factors

influencing the rate of use of community treatment orders ", Psychiatry, $\mathrm{n}^{\circ} 6$ (2), 2007.

15. Même si, en France, il es prévu dans certains cas que le préfet puisse demander que soit mis en place un programme de soins, dont le contenu reste défini par le psychiatre. 16. L. Velpry, Le quotidien de la psychiatrie, Paris, Armand Colin, 2008. 17. J. Dawson, Community Treatment Orders :

International Comparisons, Dunedin, Otago University Print, 2005, p. 19

Traduction de l'auteur. 18. D. Moreau, « Contraindre pour soigner? Le care à l'épreuve de la contrainte dans un service d'hospitalisation psychiatrique », dans C. Crignon et M. Gaille (sous la direction de), Qu'est-ce qu'un bon patient ? Qu'est-ce qu'un bon médecin? Paris, éditions Seli Arslan, 2010.

19. G. Szmukler et P. Appelbaum, « Treatment pressures, leverage, coercion, and compulsion in mental health care », Journal of Ment Health, $\mathrm{n}^{\circ} 17$ (3), juin 2008, p. 233-244.

20. J. Monahan, R.J. Bonnie, P.S. Appelbaum, P.S. Hyde,

H.J. Steadman, M.S. Swartz, «Mandated Community Treatment : Beyond Outpatient Commitment », Psychiatric Services, $\mathrm{n}^{\circ} 52$, septembre 2001, p. 1198-1205. 2001, p. T.L. Scheid-Cook,
21. «Controllers and controlled an analysis of participant constructions of outpatient commitment », Sociology of Health and Illness, $\mathrm{n}^{\circ} 15(2)$, 1993, p. 179-198. 22. G. Massé,

M. Zwingenberger,

«Les sorties d'essai :

évolution et perspectives ", L'Information psychiatrique, $n^{\circ} 82(6), 2006$

23. E.F. Torrey, M. Zdanowicz, "Outpatient Commitment : What, Why, and for Whom », Psychiatric Services, $n^{\circ} 52$, mars 2001, p. 337-341.

24. J. Dawson, Community Treatment Orders

International Comparisons, Dunedin, Otago University Print, 2005, p. 18

25. G. Vidon, « Pour des soins obligatoires en ambulatoire $x$ Nervure, VIII, $\mathrm{n}^{0}$ 5, 1995 D. Leguay, « Soins sans consentement : y a-t-il un sens de l'histoire? » Perspectives Psy, n० 48 (1), 2009 26. Loi n ${ }^{\circ} 2002-303$ du 4 mars 2002 relative aux droits des malades et à la qualité du système de santé, JORF du 5 mars 2002.
Commentant le large usage fait en Nouvelle-Zélande des mesures de soins ambulatoires sans consentement, au point qu'elles dépassent en nombre les hospitalisations sans consentement, un psychiatre estimait qu'il s'agissait là d'un processus de « désinstitutionnalisation de la législation sur la santé mentale néo-zélandaise ${ }^{24}$ ». C'est en ce sens que plusieurs psychiatres en France ont plaidé ces dernières années pour les soins ambulatoires sans consentement ${ }^{25}$. En dissociant l'hospitalisation du soin sans le consentement du patient en psychiatrie, la loi du 5 juillet 2011 offre la possibilité de reposer un certain nombre de questions éthiques et cliniques. Ces questions portent sur les conditions dans lesquelles les pratiques contraignantes sont justifiées en psychiatrie, sur la nature exacte de ces pratiques et sur les enjeux liés à leur formalisation. Plus précisément, parce qu'elle suppose un élargissement des critères ouvrant à une hospitalisation sans consentement, la mesure de soins ambulatoires sans consentement appelle à reconsidérer la définition des trois critères habituels, qui sont la dangerosité pour soi ou autrui, l'incapacité à consentir et la nécessité de soins immédiats. On a vu plus haut que la transposition des critères habituellement requis pour l'hospitalisation sans consentement amène à les adapter lorsque l'on étend la mesure hors de l'hôpital. D'une part, la mesure s'envisage dans la durée et soulève donc le problème de définir une incapacité à consentir stable dans le temps, et ce alors même que la personne peut avoir une vie relativement autonome. D'autre part, si l'on conserve le critère de dangerosité, il reste à caractériser une dangerosité qui ne nécessiterait qu'une obligation légale de soin. Du point de vue de la conception de la mesure, il est également nécessaire de définir le périmètre de ce qui relève du soin, la durée de la mesure ainsi que les conditions de sa levée. Enfin, si une obligation de suivi pèse sur le patient, il faut envisager les moyens qui permettent de s'assurer de la mise en œuvre effective de la mesure. Est-ce au législateur de déterminer à l'avance les conséquences du non-respect de la mesure, ou ces conséquences doivent-elles être fixées pour chaque patient?

Alors qu'ont été réaffirmés récemment le caractère contractuel de la relation thérapeutique et le droit au refus de traitement ${ }^{26}$, c'est donc la question des conditions dans lesquelles on peut s'autoriser à intervenir sans le consentement qui est posée dans un nouveau contexte. 